\title{
Effects of exercise on cancer-related fatigue in Pediatric Oncology: A comparison of an inpatient program at the beginning and in the middle of cancer therapy
}

\author{
Platschek AM. ${ }^{1}$, Simon T. ${ }^{2}$, Hero B. ${ }^{2}$, Foitschik T. ${ }^{1}$, Strüder HK. ${ }^{1}$
}

Deutsche

Sporthochschule Köln

German Sport University Cologne
${ }^{1}$ German Sport University Cologne, Institute of Movement and Neurosciences

${ }^{2}$ Children's Hospital, University of Cologne, Dept. Pediatric Oncology and Hematology
Objective: One of the most common side effects of cancer therapy in pediatric oncology is cancer-related fatigue [CRF] [1]. CRF is multidimensional, with the most distressing symptoms affecting quality of life [2]. The underlying mechanisms and pathophysiology are unclear, also there is still uncertainness about the potential of physical activity in the context of CRF in pediatric oncology patients. Purpose of our study was to evaluate the effects of a computer-based exercise program at the beginning versus in the middle of cancer therapy on CRF in pediatric oncology.

Materials and Methods: The computer-based exercise program at the beginning of therapy [bt] had a duration of two months and included twelve subjects (mixed cancer, age $13.3 \pm 2.5$ years, 2 females). Mean time from diagnosis to the beginning of study was $14.8 \pm 9.8$ days. The computer-based exercise program in the middle of therapy [mt] had a duration of three months and included nine subjects (mixed cancer, age $11.3 \pm 2.2$ years, 6 females). Mean time from diagnosis to the beginning of study was $58.2 \pm 62.0$ days. In both studies, exercise program was conducted via exergaming, with the same games and the possibility of supervised exercise twice a week in the inpatient hospital. Intensity and duration of each intervention session were self-selected and chosen depending on the patient's age and daily condition. The standard treatment for the pediatric patients was adjuvant chemotherapy according to national protocols. The PedsQL ${ }^{\mathrm{TM}}$ Multidimensional Fatigue Scale [3] questionnaire was completed before and after intervention. Pearson correlations were used to analyze the data ( $\alpha=0.05)$.

Results: No adverse events and no drop-outs were observed during the studies. During the $\mathrm{bt} / \mathrm{mt}$ intervention, participants performed a mean of $8.8 \pm 1.0 / 10.7 \pm 2.1$ exercise sessions with an average of $45.2 \pm 13.8 / 48.5 \pm 14.8$ minutes. Patients in bt show an overall CRF score of $67.9 \pm 10.9$ pre and $62.7 \pm 18.9$ post intervention $(p=0.288)$, higher scores indicate lower CRF. CRF related to sleep/rest was $57.3 \pm 16.3$ pre and $53.5 \pm 25.2$ post intervention ( $p=0.607$ ) (Fig. 1,2 ).
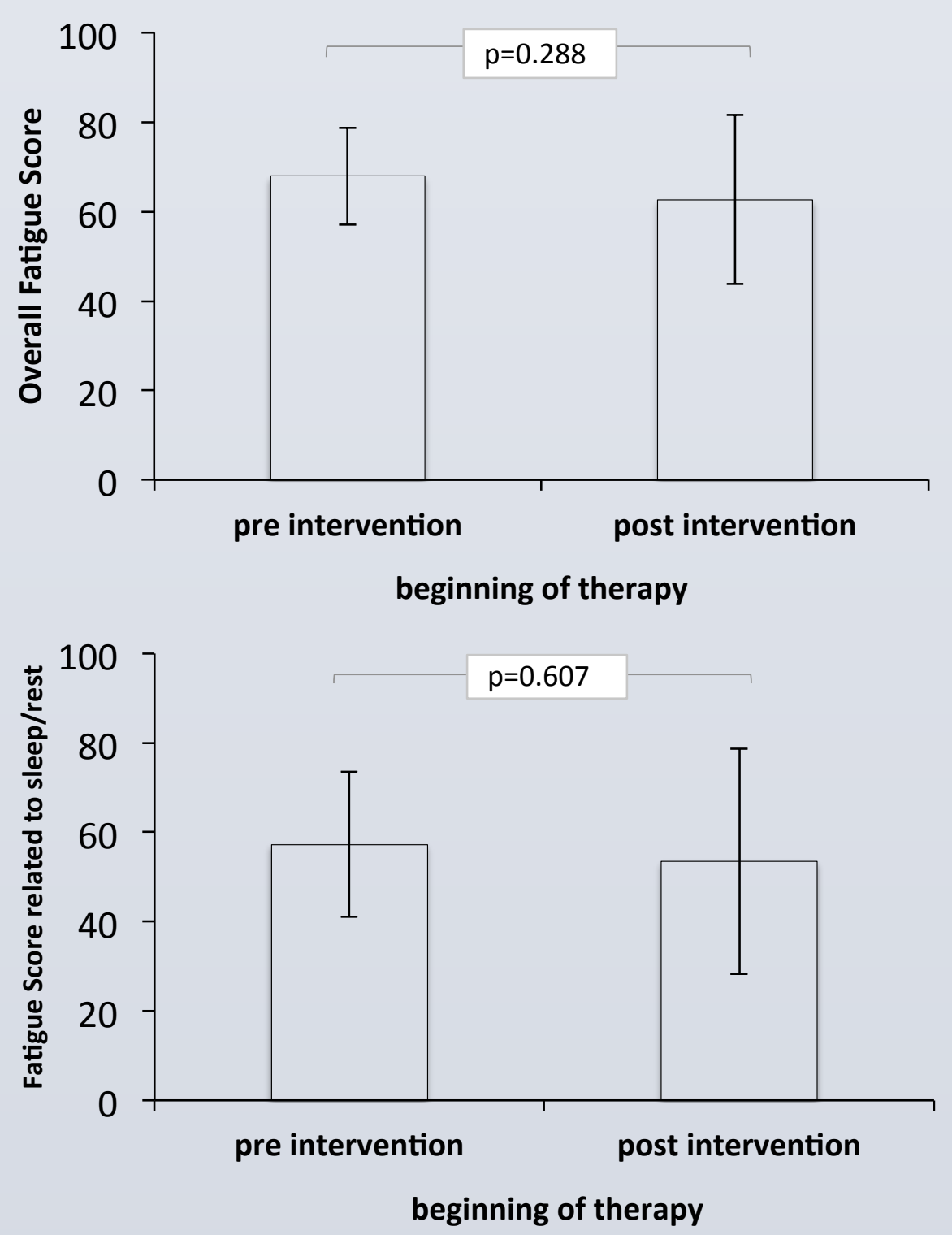

Fig. 1, 2: Fatigue Score pre and post exercise intervention at the beginning of therapy

Patients in $\mathrm{mt}$ show an overall CRF score of $66.0 \pm 17.4$ pre and $75.6 \pm 18.8$ post intervention $(p=0.127)$. CRF related to sleep/rest was $59.3 \pm 22.2$ pre and $75.9 \pm 21.1$ post intervention $(p=0.022)$ (Fig. $3,4)$.
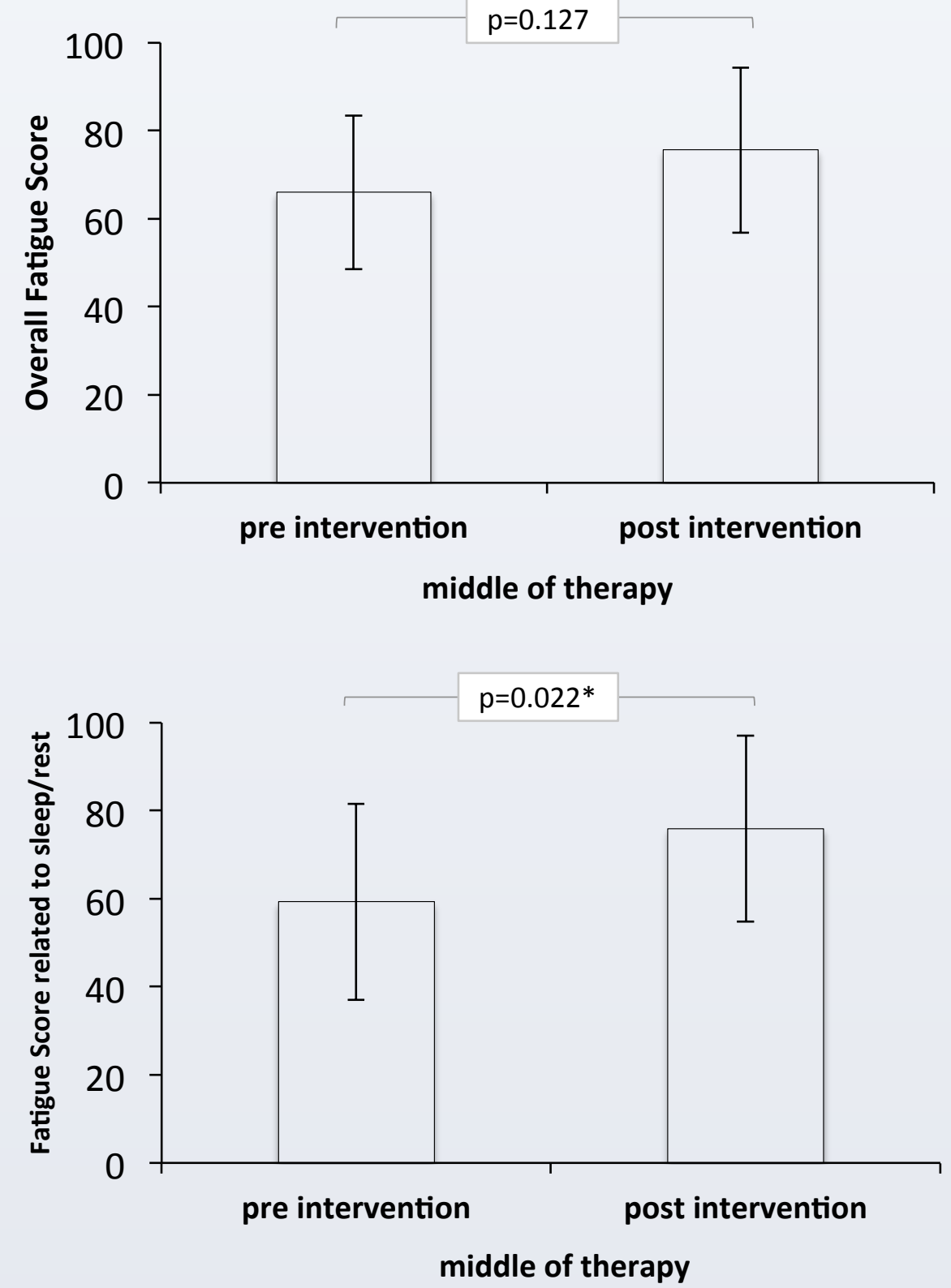

Fig. 3, 4: Fatigue Score pre and post exercise intervention in the middle of therapy

Conclusion: In pediatric oncology exercise intervention is possible at the beginning and in the middle of cancer therapy and can be integrated into everyday hospital life. Exercise intervention in the middle of cancer therapy seems to be more effective on reducing CRF than at the beginning of therapy. At that time, this result remains unclear, without being able to exclude training physiological, medical, psychological or infrastructural connections. Exergaming could be a good option for pediatric oncology patients because it is feasible for children with and without risk of infection, it is suitable for use in vulnerable populations and it offers a high fun factor. Exercise represents an effective therapeutic intervention in the middle of therapy for preparing children to successfully complete treatments, and for reducing acute side effects like CRF.

\section{References:}

[1] Baggott C, Dodd M, Kennedy C, Marina N, \& Miaskowski C (2009) Multiple symptoms in pediatric oncology patients: a systematic review. Journal of Pediatric Oncology Nursing 26(6):325-39

[2] Hofman M, Ryan JL, Figueroa-Moseley CD et al (2007) Cancer-related fatigue: the scale of the problem. The Oncologist 12(Suppl 1):4-10

[3] Varni JW, Burwinkle TM, Katz ER, Meeske K, \& Dickinson P (2002) The PedsQL in pediatric cancer: Reliability and validity of the Pediatric Quality of Life Inventory Generic Core Scales, Multidimensional Fatigue Scale, and Cancer Module. Cancer, 94, 2090-2106 